\title{
FRECUENCIA DE NEOPLASIAS EN GLÁNDULA MAMARIA DE CANINOS: ESTUDIO RETROSPECTIVO EN EL PERIODO 1992-2006 EN LA CIUDAD DE LIMA, PERÚ
}

\author{
Frequency of Neoplasms in Canine Mammary Gland: Retrospective Study in \\ the Period 1992-2006 in Lima, Peru
}

\section{Guillermo Chau V. ${ }^{1,3}$, Alfonso Chavera C. ${ }^{1}$, Rosa Perales C. ${ }^{1,4}$, César Gavidia C. ${ }^{2}$}

\section{Resumen}

\begin{abstract}
El objetivo del estudio fue determinar la frecuencia de presentación de neoplasias en la glándula mamaria de caninos, diagnosticadas histopatológicamente en el Laboratorio Patología de la Facultad de Medicina Veterinaria de la Universidad de San Marcos, Lima, a través de un estudio retrospectivo que cubrió un periodo de 13 años (1994-2006). Se tuvieron 4496 protocolos para la especie canina, de los cuales 1128 correspondieron a procesos neoplásicos $(25 \pm 1.2 \%)$. Dentro de estos, 204 casos fueron de neoplasia en glándula mamaria $(18.1 \pm 2.3 \%)$. Las neoplasias mamarias se presentaron principalmente en hembras (94\%) y en animales mestizos (38.3\%). Los caninos mayores de 10 años de edad fueron los más afectados $(46.4 \%)$. El par mamario más comprometido fue el inguinal (32.6\%). Las neoplasias malignas fueron más frecuentes (78\%) que las benignas. El adenocarcinoma fue el tipo de neoplasia más común (53.4\%).
\end{abstract}

Palabras clave: frecuencia, caninos, neoplasia, glándula mamaria

\section{Abstract}

The aim of the study was to determine the frequency of neoplasms in the mammary gland of dogs. In this retrospective study (period of 1994-2006), records from the Patology Laboratory of the Veterinary Medicine Faculty, San Marcos University were used. There were a total of 4496 canine protocols of which 1128 were corresponded to neoplasms ( 25 $\pm 1.2 \%)$. Among them, 204 cases were in the mammary gland $(18.1 \pm 2.3 \%)$. Mammary neoplasms gland mainly occurred in females (94\%) and in crossbred animals (38.3\%).

${ }^{1}$ Laboratorio de Histología, Embriología y Patología Veterinaria, ${ }^{2}$ Laboratorio de Medicina Veterinaria Preventiva, Facultad de Medicina Veterinaria, Universidad Nacional Mayor de San Marcos, Lima

${ }^{3}$ E-mail: guillermochauv@gmail.com

${ }^{4}$ E-mail: rperales_fmv@hotmail.com 


\begin{abstract}
Dogs older than 10 years of age were most affected (46.4\%). The inguinal pair was also the most affected (32.6\%). Malignant neoplasms were more frequent $(78 \%)$ than benign neoplasms. The adenocarcinoma was the most common neoplasm affecting the mammary gland $(53.4 \%)$.
\end{abstract}

Key words: frequency, canine, neoplasm, mammary gland

\section{INTRODUCCIÓN}

El creciente interés de la sociedad por el bienestar animal ha causado una mayor demanda y preocupación por parte de los dueños de mascotas en el diagnóstico y tratamiento de las enfermedades que afectan a sus animales; además, el avance científico de la Medicina Veterinaria ha permitido mejorar las técnicas de diagnóstico de laboratorio y la creación de laboratorios especializados de diagnóstico.

El diagnóstico de procesos neoplásicos es de gran importancia para la práctica clínica de la Medicina Veterinaria, particularmente de aquellos que afectan a los caninos. La frecuencia de tumores en esta especie es frecuente y, comparativamente, las neoplasias que afectan la glándula mamaria ocupan el segundo lugar en frecuencia, por detrás de aquellas que se presentan en la piel (Jones y Hunt, 1990; Moulton, 1990; Mangieri et al., 1991; Benjamín et al., 1999). Las neoplasias mamarias tienen su origen en cualquiera de los tejidos adyacentes de la glándula mamaria, siendo el tumor mamario mixto el más común (Jennings, 1975).

Las neoplasias de glándula mamaria en el canino representan el $13 \%$ de todas las neoplasias caninas y el $50 \%$ de las que se presentan en la perra (Rutteman et al., 2001; Sorenmo, 2003). Takano (1964) señaló que las neoplasias de glándula mamaria en perras eran el tipo más frecuentes de neoplasias $(28 \%)$. Asimismo, se menciona una incidencia de $0.2 \%$ de neoplasias mamarias en poblaciones de perras (Doliger, 2003). Estudios retrospectivos llevados a cabo en Colombia sobre 136 casos de tumores mamarios en perras encontraron 58\% de carcinomas, $23 \%$ de tumores mixtos benignos y $9.5 \%$ de tumores mixtos malignos (Ferreira de la Cuesta y Pedraza, 2003; Torres, 2003). Finalmente, se menciona que la frecuencia de neoplasias en glándula mamaria de la perra es es tres veces superior que en la mujer (Schneider, 1970).

El Laboratorio de Patología de la Facultad de Medicina Veterinaria de la Universidad Nacional Mayor de San Marcos (FMVUNMSM) es un centro referencial para casos oncológicos provenientes de las clínicas y consultorios de mascotas de la ciudad de Lima con la finalidad de confirmar sus diagnósticos clínicos. El presente estudio tuvo como objetivo analizar la frecuencia de casos de neoplasias mamarias de caninos, así como las variables que afectan su presentación.

\section{Materiales y Métodos}

Se trabajó con los registros de diagnósticos histopatológicos de biopsias y necropsias de caninos del Laboratorio de Patología Veterinaria de la FMV-UNMSM, que cubrían el periodo de enero de 1994 a diciembre de 2006.

Se registró la información correspondiente a edad $(<1 ; \geq 1$ y $<6 ; \geq 6$ y $<10 ; \geq 10$ años), sexo (macho; hembra), raza (incluyendo los cruzados o de raza no definida), diagnóstico histopatológico y localización de las neoplasias. 
Para el análisis de la localización de la neoplasia, se consideró las formaciones neoplásicas en cada uno de los pares mamarios, así como el lado de aparición (derecho o izquierdo). La clasificación histológica de las neoplasias de la glándula mamaria se hizo considerando el grado de malignidad. La frecuencia de neoplasias se presentó en forma porcentual con su respectivo intervalo de confianza al 95\%, haciendo mención especial a los casos de neoplasias mamarias. En el análisis estadístico se utilizó la prueba de Chi cuadrado.

\section{Resultados}

Se encontró un total de 4496 protocolos en caninos correspondientes al periodo de 13 años de estudio, de los cuales 1128 correspondieron a procesos neoplásicos $(25.1 \%)$ y 204 casos a neoplasias en glándula mamaria $(4.5 \%)$. Los resultados demuestran una frecuencia de $18.1 \%$ de neoplasias mamarias dentro del total de procesos neoplásicos.

Datos sobre el sexo se obtuvo en 199 protocolos de los 204 diagnósticos de neoplasia mamaria, correspondiendo el $94 \%$ de casos para las hembras y $6 \%$ para los machos $(\mathrm{p}<0.05)$. Datos sobre la raza estaban consignados en 193 casos, encontrándose que la mayor frecuencia de casos se presentó en perras mestizas (38.3\%), seguido por la raza Cocker (13\%) y otras (Cuadro 1).

Las neoplasias mamarias se observaron con mayor frecuencia en perras mayores de 10 años de edad $(46.4 \pm 7 \%, 90 / 194)$, seguido de perras entre 6 y 10 años de edad con $41.8 \pm 7 \%$ (81/194) y perras entre 1 a 6 años de edad con $11.8 \pm 4.6 \%$ (23/194). No se observaron casos en hembras menores de un año de edad.

En el Cuadro 2 se observa la distribución de las neoplasias según su localización. El par inguinal presenta la mayor frecuencia
Cuadro 1. Frecuencia de neoplasias mamarias caninas según la raza

\begin{tabular}{lcc}
\hline Raza & N. $^{\circ}$ & $\% \pm$ I.C. $^{1}$ \\
\hline Mestizos & 74 & $38.3 \pm 6.9$ \\
Cocker & 25 & $13.0 \pm 4.7$ \\
Boxer & 12 & $6.2 \pm 3.4$ \\
Labrador Retriever & 11 & $5.7 \pm 3.3$ \\
Pastor Alemán & 10 & $5.2 \pm 3.1$ \\
Poodle & 7 & $3.6 \pm 2.6$ \\
Rottweiler & 7 & $3.6 \pm 2.6$ \\
Yorkshire & 7 & $3.6 \pm 2.6$ \\
Pekinés & 6 & $3.1 \pm 2.4$ \\
Dobermann & 5 & $2.6 \pm 2.2$ \\
Samoyedo & 4 & $2.1 \pm 2.0$ \\
Fox Terrier & 3 & $1.6 \pm 1.7$ \\
Siberian Husky & 3 & $1.6 \pm 1.7$ \\
Dogo & 2 & $1.0 \pm 1.4$ \\
Otros & 17 & $8.8 \pm 4.6$ \\
\hline Total & 193 & 100.0 \\
\hline
\end{tabular}

${ }^{1}$ Intervalo de confianza del $95 \%$

$(32.6 \pm 8.0 \%)$. Algunos animales presentaron neoplasias en más de un par mamario y 121 animales no fueron incluidos en el análisis por no presentar datos relativos a la localización de la neoplasia.

Las neoplasias malignas $(\mathrm{n}=163)$ predominaron sobre las benignas $(\mathrm{n}=45)$ con un $78.0 \pm 5.6 \%(163 / 208)$ sobre $22.0 \pm 5.6 \%$ (45/208). Dentro de las neoplasias malignas, predominó el adenocarcinoma con $53.4 \pm$ $6.8 \%$ (111/208), seguido del tumor mamario mixto maligno con $22.1 \pm 5.6 \%$ (Fig. 1). En el caso de las neoplasias mamarias benignas, el adenoma fue el de mayor frecuencia con $5.3 \pm 2.9 \%$ (11/208), seguido del tumor mamario mixto benigno $(4.3 \pm 2.7 \%, 9 / 208)$, el fibroadenoma $(0.5 \pm 0.9 \%, 1 / 208)$, y otros de menor importancia $(11.5 \pm 4.4 \%, 24 / 208)$. 
Cuadro 2. Frecuencia de neoplasias mamarias en el canino según su localización anatómica $(\mathrm{n}=83$ canes $)$

\begin{tabular}{lcccccc}
\hline \multirow{2}{*}{ Glándulas mamarias } & \multicolumn{3}{c}{ Lado derecho } & \multicolumn{2}{c}{ Lado izquierdo } & \multicolumn{2}{c}{ Total } \\
\cline { 2 - 7 } & $\mathrm{N} .^{\circ}$ & $\%$ & $\mathrm{~N} .^{\circ}$ & $\%$ & $\mathrm{~N} .{ }^{\circ}$ & $\%$ \\
\hline Torácicas craneales & 6 & $4.6 \pm 3.8$ & 10 & $7.8 \pm 4.7$ & 16 & $12.4 \pm 5.6$ \\
Torácicas caudales & 4 & $3.1 \pm 2.9$ & 9 & $6.9 \pm 4.4$ & 13 & $10.1 \pm 5.2$ \\
Abdominales craneales & 6 & $4.6 \pm 3.8$ & 18 & $13.9 \pm 5.9$ & 24 & $18.6 \pm 6.6$ \\
Abdominales caudales & 19 & $14.7 \pm 6.2$ & 15 & $11.6 \pm 5.6$ & 34 & $26.4 \pm 7.6$ \\
Inguinales & 21 & $16.3 \pm 6.3$ & 21 & $16.3 \pm 6.3$ & 42 & $32.6 \pm 8.0$ \\
\hline Total de focos & 56 & & 73 & & \multirow{2}{*}{129} & \\
neoplásicos & 56 & & & & & \\
\hline
\end{tabular}

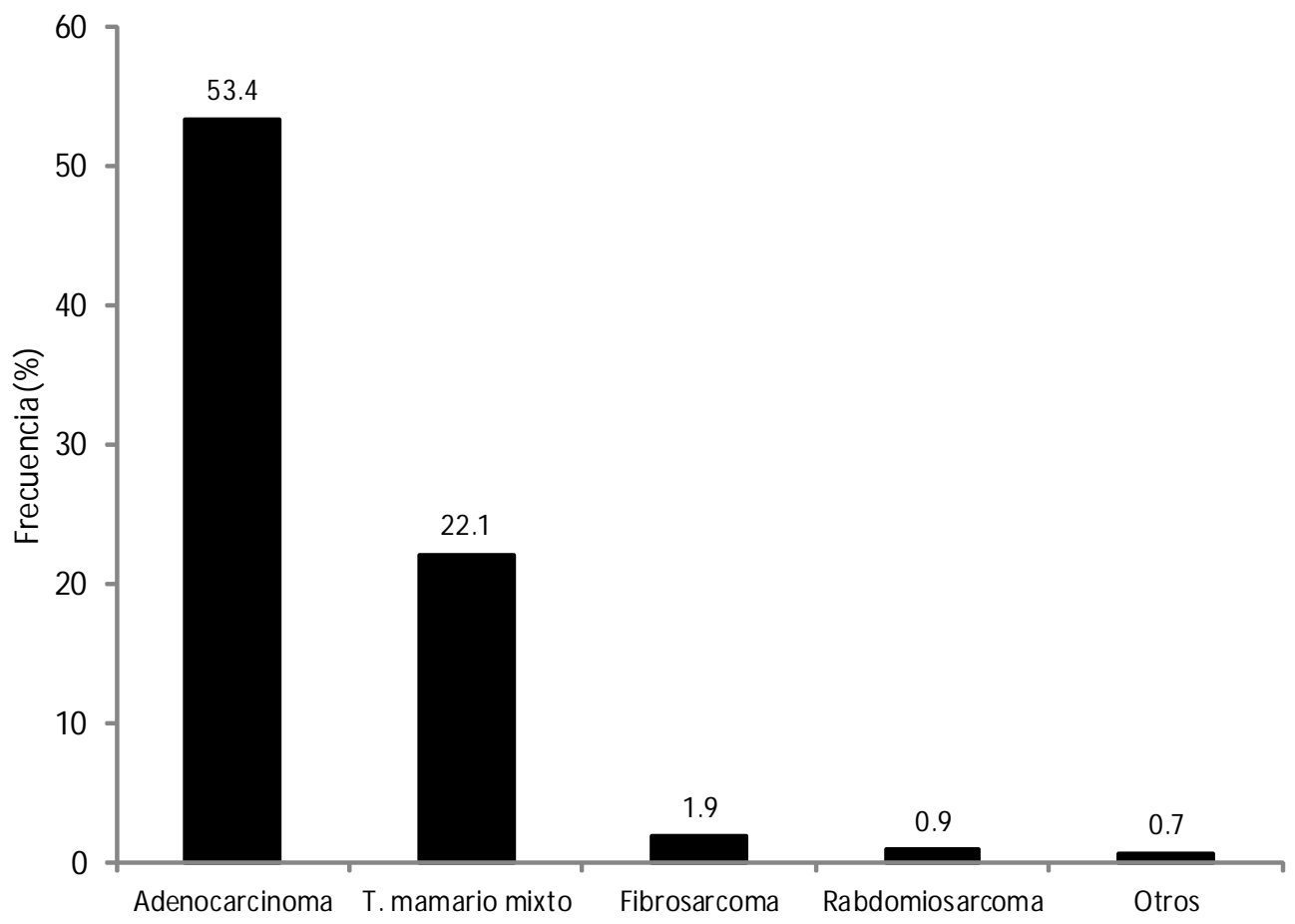

Figura 1. Frecuencia de presentación de los tipos de neoplasias malignas en glándula mamaria 


\section{Discusión}

El $25.1 \%$ de frecuencia de casos de neoplasias caninas fue muy similar al $28 \%$ reportado por Takano para muestras del mismo laboratorio en el periodo 1948-1963. Por otro lado, la mayor frecuencia de neoplasias en hembras que en machos fue muy notoria, coincidiendo con otros estudios (Jubb et al., 1991; Orbegoso, 1993; Rostami et al., 1994).

La mayor frecuencia de neoplasias mamarias en canes mestizos es en concordancia con la mayor frecuencia de este tipo de animales en la ciudad de Lima, tal y como ocurre en otras ciudades latinoamericanas (Ferreira de la Cuesta y Pedraza, 2003).

El 78\% de neoplasias malignas fue muy superior a los valores de otros estudios donde se encuentran niveles similares entre neoplasias malignas y benignas (Brodey et al., 1983; Meuten, 2002; Doliger, 2003; Novasad, 2003); sin embargo, esta diferencia puede estar dada por el menor interés de las clínicas veterinarias en Lima de remitir muestras al laboratorio de tejidos neoplásicos aparentemente benignos. Asimismo, se observan notables diferencias con estudios realizados en otras ciudades del continente; así, en el estudio de Ferreira de la Cuesta y Pedraza (2003) se reporta una mayor frecuencia de carcinomas (58\%), seguido del tumor mamario mixto benigno (23\%).

Los resultados por efecto del grupo etario concuerdan con otros reportes, donde se observa una fuerte asociación entre la edad avanzada y el mayor nivel de presentación de neoplasias mamarias. En el presente estudio se encontró la mayor frecuencia en perras de 10 o más años de edad (46.4\%), en tanto que Takano (1964) señala una mayor frecuencia entre los 7 y 8 años, Moulton (1978) entre 6 a 11 años, Rodríguez (1978) entre 3 a 12 años, y Díaz (1990) indica 8 años como la edad con mayor prevalencia de tumores mamarios. Una de las razones de la mayor frecuencia de neoplasias mamarias en perras adultas y viejas es debido a que las células mamarias han pasado por más ciclos celulares y han estado expuestos por mayor tiempo a factores carcinógenos (Jones et al., 1990; Owen, 1991).

La frecuencia de distribución de neoplasias en los pares mamarios coincide con otros reportes de la literatura que señalan que las mamas torácicas craneales son las menos afectadas y que los dos últimos pares son los más afectados (Withrow, 1975; Jubb et al., 1991).

\section{Conclusiones}

- El $18.1 \%$ de neoplasias en glándula mamaria representa un porcentaje importante en relación al total de neoplasias en caninos.

- La mayor frecuencia de neoplasias mamarias se presentó en hembras (94\% $\pm 3.3 \%$ ); asimismo, la frecuencia de presentación aumentó conforme se incrementó la edad, siendo el grupo igual o mayor de 10 años el más afectado $(46.4 \%)$.

- Las neoplasias mamarias malignas (78\%) predominaron sobre las benignas (22\%). Dentro de las neoplasias malignas, los casos de adenocarcinoma (53.4\%) fueron los más frecuentes.

\section{Literatura Citada}

1. Benjamín SA, Lee AC, Saunders WJ. 1999. Classification and behavior of canine mammary epithelial neoplasm based on lifespan observations in Beagles. Vet Pathol 36: 423-436.

2. Brodey RS, Goldschmidt MH, Roszel JR. 1983. Canine mammary gland neoplasms. J Am Anim Hosp Assoc 19: 61-90.

3. Díaz M. 1991. Procesos neoplásicos en caninos, análisis estadístico, periodo 1984-1989. Tesis de Médico Veterina- 
rio. Lima: Facultad de Medicina Veterinaria, Universidad Nac Mayor de San Marcos. 28 p.

4. Doliger S. 2003. Vade-Mecum de cancérologie Vétérinaire. París: Éditions Med'com. 224 p.

5. Ferreira De La Cuesta G, Pedraza F. 2003. Caracterización y análisis de las neoplasias registradas en el laboratorio de patología animal de la Universidad de Antioquia durante 30 años (1968-1998). En: Ferreira de La Cuesta G (ed). Patología veterinaria. Colombia: Ed Universidad de Antioquia. 573 p.

6. Jennings AR. 1975. Patología animal. México: Ed La Prensa Médica Mexicana. $295 \mathrm{p}$.

7. Jones TC, Hunt RD. 1990. Patología veterinaria. $2^{\mathrm{a}}$ ed. Buenos Aires: Ed Hemisferio Sur. $276 \mathrm{p}$.

8. Jubb KVF, Kennedy PCK, Palmer N. 1991. Patología de los animales domésticos. $3^{\circ}$ ed. Tomo III. Montevideo: Hemisferio Sur. $571 \mathrm{p}$.

9. Mangieri J, Ciraolo J, Gómez H, Pajot S. 1991. Incidencia de las neoplasias mamarias y algunos supuestos factores predisponentes en caninos. En: Jornadas de Actualización Veterinaria. Colegio Médico Veterinario de la Provincia de Santa Fe, Argentina.

10. Meuten JD. 2002. Tumors in domestic animals. $4^{\text {th }}$ ed. USA: Iowa State Press. $788 \mathrm{p}$.

11. Moulton J. 1978. Tumors in domestic animals. $2^{\text {nd }}$ ed. Berkeley, USA: California Press. 465 p.

12. Moulton JE. 1990. Tumors in domestic animals. $3^{\text {rd }}$ ed. Berkeley, USA: California Press. 472 p.

13. Novasad AC. 2003. Principles of treatment for mammary gland tumors. Clin Tech Small An P 18: 107-109.

14. Orbegoso G 1993. Neoplasias en glándula mamaria de caninos: estudio estadístico, periodo 1973-1992. Tesis de Médico Veterinario. Lima: Facultad de
Medicina Veterinaria, Universidad Nac Mayor de San Marcos. 40 p.

15. Owen $L N$. 1991. Identifying and treating cancer in geriatric dogs. Vet Med 86: 55-64.

16. Rodriguez G. 1978. Neoplasias en la especie canina, estudio estadístico de 301 casos entre 1964-1977. Lima: Tesis de Médico Veterinario Facultad de Medicina Veterinaria, Universidad Nac Mayor de San Marcos. 35 p.

17. Rostami M, Tate Y, Uchida K, Naitou H, Yamaguchi R, Otsuka H. 1994. Tumors in domestic animals examined during a ten year period (1980-1989) at Miyazaki University. J Vet Med 56: 403405.

18. Rutteman GR, Withrow SJ, Macewen EG. 2001. Tumors of the mammary glands. In: Small Animal Clinical Oncology. $3^{\text {rd }}$ ed. USA: WB Saunders. p 455-477.

19. Schneider R. 1970. Comparison of age, sex and incidence rates in human and canine breast cancer. Cancer 26: 419426.

20. Sorenmo K. 2003. Canine mammary gland tumors. Vet Clin N Am Small 33: 573-596.

21. Takano F. 1964. Estudio estadístico de 154 procesos neoplásicos en la especie canina. Casos registrados en el Departamento de Patología de la Facultad de Medicina Veterinaria en el periodo comprendido entre los años 1948-1963. Tesis de Médico Veterinario. Lima: Facultad de Medicina Veterinaria, Universidad Nac Mayor de San Marcos. 55 p.

22. Torres VG. 2003. Estudio histológico retrospectivo de la casuística de tumores mamarios caninos diagnosticados en el laboratorio de patología de la Facultad de Medicina Veterinaria y Zootecnia de la Universidad Nacional de Colombia. Tesis de especialización. Antioquía: Universidad Nacional de Colombia. 13 p.

23. Withrow S. 1975. Surgical management of canine mammary tumors. Vet Clin N Am Small 5: 495-506. 